\title{
CONSTITUINTES QUÍMICOS E ATIVIDADE ANTIOXIDANTE DE Sida galheirensiS ULBR. (MALVACEAE)
}

\author{
Davi Antas e Silva, Tânia Maria Sarmento da Silva, Antônio Cláudio da Silva Lins, Danielly Albuquerque da Costa, \\ José Marcílio Sobral Cavalcante, Wemerson Neves Matias e Maria de Fátima Vanderlei de Souza* \\ Laboratório de Tecnologia Farmacêutica "Delby Fernandes de Medeiros", Universidade Federal da Paraíba, \\ CP 5009, 58051-970 João Pessoa - PB, Brasil
}

Raimundo Braz Filho

Setor de Química de Produtos Naturais, Centro de Ciências e Tecnologia, Universidade Estadual do Norte Fluminense, Av. Alberto Lamego, 2000, 28013-603 Campos - RJ, Brasil

Recebido em 6/12/05; aceito em 9/3/06; publicado na web em 11/8/06

\begin{abstract}
CHEMICAL CONSTITUENTS AND ANTIOXIDANT ACTIVITY OF Sida galheirensis Ulbr. (Malvaceae). The phytochemical investigation of Sida galheirensis led to the isolation of 5,4'-dihydroxy-3,7,3'-trimethoxyflavone, $17^{3}$-ethoxyphaeoforbide, a rare natural product, 6,7-dimethoxycoumarin, ortho-hydroxybenzoic acid, sitosterol-3- $O-\beta$-D-glucopyranoside, stigmasterol-3- $O-\beta$-D-glucopyranoside, $5,7,4$ '-trihydroxyflavone, 5,7,3',4'-tetrahydroxyflavone, kaempferol-3- $O$ - $\beta$-D-(6"-E- $p$-coumaroyl) glucopyranoside and luteolin 7-O$\beta$-D-glucopyranoside. Their structures were assigned based on spectroscopic analyses, including two-dimensional NMR techniques. Antioxidant activities of hexane, $\mathrm{CHCl}_{3}, \mathrm{EtOAc}, \mathrm{BuOH}$ and $\mathrm{EtOH}$ extracts of Sida galheirensis were measured using the 1,2-diphenyl2-picryl-hydrazyl (DPPH) free radical scavenging assay. This is also the first work reporting the chemical investigation of Sida galheirensis.
\end{abstract}

Keywords: Sida galheirensis; Malvaceae; flavonoids.

\section{INTRODUÇÃO}

O gênero Sida apresenta ampla distribuição neotropical com várias espécies bem representadas nas Américas. No Brasil este gênero possui muitos representantes nas regiões Nordeste e Sul e, em menor proporção, nas regiões Norte, Centro-Oeste e Sudeste ${ }^{1}$.

Espécies de Sida são usadas na medicina popular com diversas atividades, como a Sida acuta, empregada para neutralizar o veneno da serpente Bothrops atrox, tendo esse efeito sido investigado em laboratório ${ }^{2}$. A Sida cordifolia, conhecida como malva branca, é usada na medicina folclórica para tratamento de estomatites, bronquite asmática e congestão nasal. Os efeitos antiinflamatório e analgésico foram investigados para o extrato aquoso desta planta, mostrando-se bastante significativos, comprovando sua utilização popular $^{3}$. A Sida rhomboidea Roxb é uma erva encontrada em pântanos da Índia, cujas raízes e folhas são usadas como tônico, para cura de febres, doenças do coração e todos os tipos de inflamação. Estudos farmacológicos utilizando extratos desta planta indicaram sua atividade antinociceptiva e antiinflamatória ${ }^{4}$.

Investigações fitoquímicas com espécies do gênero Sida mostraram a presença de ácidos $\operatorname{graxos}^{5-9}$, diterpenos ${ }^{7}$, esteróides ${ }^{10,11}$, flavonóides ${ }^{12,13}$ e compostos nitrogenados ${ }^{14,15}$.

Os radicais livres desempenham papel importante no organismo, mas o efeito cumulativo desses radicais está implicado em doenças, tais como câncer, ateroscleroses, isquemia cerebral e envelhecimento ${ }^{16}$. Antioxidantes que seqüestram os radicais livres, tanto previnem como apresentam alto potencial terapêutico em doenças que apresentam estes radicais ${ }^{17,18}$. Estas observações levam à busca de novos potenciais antioxidantes derivados de plantas utilizadas na medicina popular.

*e-mail: mfvanderlei@ltf.ufpb.br
Este trabalho descreve o estudo fitoquímico e a avaliação da atividade antioxidante de Sida galheirensis, uma espécie com endemismo em regiões do semi-árido, ocorrendo em praticamente todos os estados do Nordeste, normalmente em densas populações em meio à caatinga ${ }^{1}$.

\section{PARTE EXPERIMENTAL}

\section{Procedimentos experimentais gerais}

Os espectros de absorção na região do infravermelho foram obtidos em aparelho Perkin-Elmer, FT-IR-1750 utilizando-se 3,0 $\mathrm{mg}$ de amostra em pastilhas de $\mathrm{KBr}$, com freqüência medida em $\mathrm{cm}^{-1}$. Os espectros de RMN foram obtidos em espectrômetros Brucker-AC (UFC) a $500\left({ }^{1} \mathrm{H}\right)$ e $125\left({ }^{13} \mathrm{C}\right)$ e Mercury-Varian a 200 $\left({ }^{1} \mathrm{H}\right)$ e $50\left({ }^{13} \mathrm{C}\right)$ (LTF/UFPB), otimizados para técnicas uni e bidimensionais, utilizando-se quantidades variáveis de amostras. Os solventes empregados foram $\mathrm{CDCl}_{3}, \mathrm{CD}_{3} \mathrm{OD}, \mathrm{C}_{6} \mathrm{D}_{5} \mathrm{~N}$ e DMSO$d_{6}$, cujos picos característicos em $\mathrm{RMN}{ }^{1} \mathrm{H}$ e ${ }^{13} \mathrm{C}$ serviram como padrão interno durante a obtenção dos espectros. Para as cromatografias em coluna utilizou-se como fase estacionária sílica gel 60 (Merck) 7734 (partículas com 0,063-0,2 mm, 70-230 mesh) e Sephadex LH-20 (Pharmacia, Uppsala-Sweden), tendo como suporte colunas de vidro cilíndricas com dimensões variando de acordo com a quantidade de amostra a ser cromatografada. A cromatografia em camada delgada comparativa (CCDC) foi empregada para análise e reunião das frações obtidas por cromatografia em coluna. Foram utilizadas placas de vidro cuja fase fixa foi preparada com uma suspensão de sílica gel $\mathrm{PF}_{254} 7749$ (Merck) em água. As substâncias em análise foram evidenciadas pelo uso de radiação ultravioleta sob os comprimentos de onda de 254 e 366 $\mathrm{nm}$, pela impregnação das placas em cubas de vidro saturadas por vapores de iodo e, ainda, com solução de $\mathrm{AlCl}_{3}: \mathrm{EtOH}$ (1\%) (para os flavonóides glicosilados). 


\section{Material vegetal}

A planta total, Sida galheirensis, foi coletada no município de Serra Branca, estado da Paraíba, em abril de 2000 e identificada pela botânica Prof ${ }^{a}$ Dra. M. de F. Agra, do Núcleo de Pesquisas em Produtos Naturais/LTF/UFPB e uma exsicata do material ( $n^{\circ} 2249$ ) foi arquivada no Herbário Lauro Pires Xavier (CCEN/UFPB).

\section{Extração e isolamento dos constituintes químicos}

O material botânico desidratado e moído (15,0 kg) foi macerado em etanol a $95 \%$ por $72 \mathrm{~h}$, sendo tal processo repetido exaustivamente. A solução etanólica foi concentrada em evaporador rotativo a $60{ }^{\circ} \mathrm{C}$, produzindo $612,0 \mathrm{~g}$ do extrato etanólico bruto. Uma parte deste material $(450,0 \mathrm{~g}$ ) foi submetida a estudo. Este material foi solubilizado em $400 \mathrm{~mL}$ de etanol:água (7:3) e extraído sucessivamente com hexano, $\mathrm{CHCl}_{3}$, AcOEt e $n$-BuOH. Após evaporação do solvente de todas as fases em rotavapor, obteve-se $111,1 \mathrm{~g}$ da fase hexânica, 76,8 $\mathrm{g}$ da fase $\mathrm{CHCl}_{3}, 13,1 \mathrm{~g}$ da fase AcOEt e 10,0 $\mathrm{g}$ da fase $n-\mathrm{BuOH}$. A fase hidroalcoólica remanescente foi filtrada para separação de um precipitado que se formou, o qual foi posteriormente cromatografado.

A fase clorofórmica $(20,0 \mathrm{~g})$ foi submetida à cromatografia em coluna de sílica gel 60 eluindo-se com hexano, AcOEt e $\mathrm{MeOH}$, seguindo uma ordem crescente de polaridade. As amostras obtidas através deste procedimento foram, então, analisadas em CCDC e reunidas de acordo com seus Rfs, resultando em 31 frações. A fração reunida 60/65 (108,0 mg) mostrou-se como duas manchas por análise em CCDC e uma alíquota de $65,0 \mathrm{mg}$ foi cromatografada em coluna com Sephadex LH-20, sendo utilizada uma mistura $\mathrm{CHCl}_{3}: \mathrm{MeOH}$ (1:1) como eluente, obtendo-se a 5,4'-diidroxi-3,73'-trimetoxiflavona (pachypodol) (1, 11,0 mg) e o $17^{3}$ etoxifaeoforbídeo a $(\mathbf{2}, 25,0 \mathrm{mg})$. A fração 84/89 forneceu a 6,7dimetoxicumarina (escoparona, 3, 65,0 mg) após recristalização em $\mathrm{CHCl}_{3}$ e a fração 103/120, após ser recromatografada em coluna de sílica gel, permitiu o isolamento do ácido orto-hidroxibenzóico $(4,23,0 \mathrm{mg})$. Uma pequena amostra da fração 210/218 $(35,0 \mathrm{mg})$ forneceu uma mistura de sitosterol-3- $O-\beta$-D-glicopiranosídeo e estigmasterol-3-O- $\beta$-D-glicopiranosídeo (5 e 6, respectivamente, $30,0 \mathrm{mg}$ ) através de recristalização com $\mathrm{MeOH}$.

A fase AcOEt (10,0 g) foi submetida à coluna de sílica gel com hexano, AcOEt e $\mathrm{MeOH}$ como eluentes em ordem crescente de polaridade. As frações obtidas foram comparadas por CCDC e reunidas, obtendo-se 18 frações. A 43/72 foi cromatografada em Sephadex LH-20, utilizando-se $\mathrm{MeOH}$ e $\mathrm{MeOH}: \mathrm{CHCl}_{3}$ (7:3), resultando na obtenção da 5,7,4'-triidroxiflavona (apigenina, 7, 15,0 mg). As frações 85/96 e 186/218 foram recristalizadas com $\mathrm{MeOH}$ e forneceram a 5,7,3',4'-tetraidroxiflavona (luteolina, $8,50,0 \mathrm{mg}$ ) e o canferol-3-O- $\beta$-D-(6"-E-p-cumaroil) glicopiranosídeo (tilirosídeo, 9, 400,0 mg), respectivamente.

Uma parte (1,5 g) do precipitado obtido da fase hidroalcoólica foi cromatografada em coluna de Sephadex LH-20, usando-se $\mathrm{MeOH}$ como eluente. As frações obtidas foram comparadas por CCDC através de visualização em luz ultravioleta e revelados com $\mathrm{AlCl}_{3} 1 \%$, sendo reunidas as semelhantes. Das 6 frações resultantes, a fração 13-15 foi recristalizada com $\mathrm{MeOH}$ para fornecer a luteolina-7-glicosídeo (cinarosídeo, 10, 80,0 mg).

5,4'-Diidroxi-3,7-3'-trimetoxiflavona (1, pachypodol). Sólido amorfo: $\mathrm{RMN}^{1} \mathrm{H}\left[500 \mathrm{MHz}, \mathrm{CDCl}_{3}, \delta(\mathrm{ppm}), J(\mathrm{~Hz})\right]:$ 7,73 (d, 1,9 Hz, H-2'), 7,69 (dd, 8,4 e 1,9 Hz, H-6'), 7,06 (d, 8,4 Hz, H-5'), 6,47 (d, 2,1 Hz, H-8), 6,38 (d, 2,1 Hz, H-6), 3,88 (s, MeO-3), 3,90 (s, MeO-7), 4,00 (s, MeO-3'). RMN ${ }^{13} \mathrm{C}\left[\left(125 \mathrm{MHz}, \mathrm{CDCl}_{3}, \delta\right.\right.$ (ppm)]: 179,15 (C-4), 165,85 (C-7), 162,43 (C-5), 157,13 (C-9), 156,34 (C-2), 148,73 (C-4'), 146,74 (C-3'), 139,26 (C-3), 123,09, (CH-6'), 122,88 (C-1'), 114,98 (CH-5'), 111,29 (CH-2'), 106,44 (C-10), 98,26 (CH-6), 92,60 (CH-8), 60,59 (MeO-3), 56,52 (MeO3'), 56,23 (MeO-7).<smiles>COc1cc(O)c2c(=O)c(OC)c(-c3ccc(Cl)c(OC)c3)oc2c1</smiles>

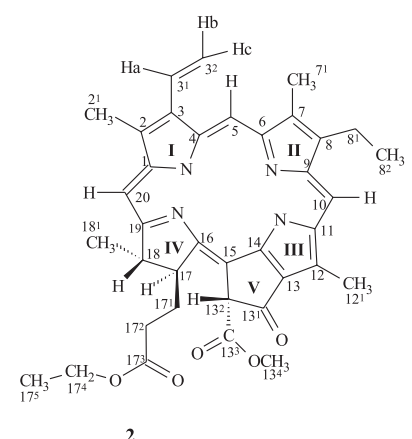<smiles>COc1cc2ccc(=O)oc2cc1OC</smiles><smiles>COc1ccccc1O</smiles>

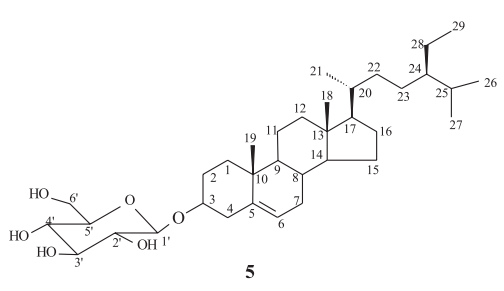

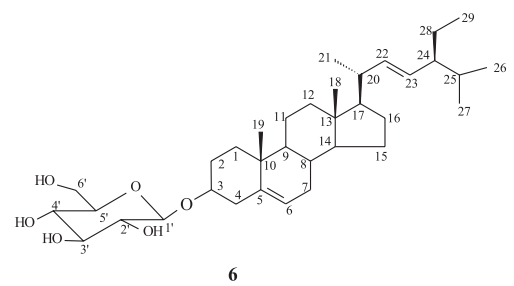<smiles>O=c1cc(-c2ccc(O)cc2)oc2cc(O)cc(Cl)c12</smiles><smiles>O=c1cc(-c2ccc(Cl)c(O)c2)oc2cc(O)cc(Cl)c12</smiles><smiles>CC1(C)CCCC1(C)OCOc1c(-c2ccc(O)cc2)oc2cc(O)cc(O)c2c1=O</smiles>

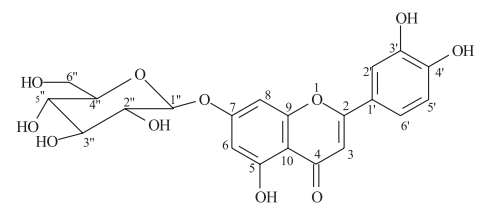

Figura 1. Constituintes químicos isolados de Sida galheirensis 
173-Etoxifaeoforbídeo a (2). Sólido cristalino: ES-MS $\mathrm{m} / z$ : 621,19 $\left(\mathrm{M}^{+\bullet}+\mathrm{H}\right), 593,23,561,24$ (100), 533,21, 473,19, 461,19. IV (KBr) $\mathrm{cm}^{-1}: 3433,2957,2908,2859,1731,1695,1612,1459,1447,1344$, $1222,1173,1025,978,896,808,669$. RMN ${ }^{1} \mathrm{H}$ e ${ }^{13} \mathrm{C}$ (Tabela 1).

Canferol-3-O- $\beta$-D-(6"-E-p-cumaroil) glicopiranosídeo (9, tilirosídeo). Pó amorfo: $\mathrm{RMN}{ }^{1} \mathrm{H}\left[200 \mathrm{MHz}, \mathrm{CDCl}_{3}, \delta\right.$ (ppm), $J$ (Hz)]: 7,96 (d, 9,0 Hz, H-2'/6'), 7,38 (d, 15,9 Hz, H- $\beta), ~ 7,25 ~(d$, 8,6 Hz, H-2'"'/6'"), 6,79 (d, 9,0 Hz, H-3'/5'), 6,77 (d, 8,6 Hz, H3"'/5'"), 6,27 (d, 2,0 Hz, H-8), 6,11 (d, 2,0 Hz, H-6), 6,05 (d, 15,9 $\mathrm{Hz}, \mathrm{H}-\alpha), 5,23$ (d, 7,6 Hz, H-1"), 4,19 (dd, 11,8 e 2,2 Hz, H-6”), 4,06 (dd, 11,6 e 6,4 Hz, H-6") 3,38-3,34 (m, H-2", 3", 4"), 3,253,16 (m, H-5”). RMN ${ }^{13} \mathrm{C}\left(50 \mathrm{MHz}, \mathrm{CDCl}_{3}, \delta\right.$ (ppm)]: 179,30 (C4), 168,82 (COO), 165,79 (C-7), 162,82 (C-5), 161,43 (C-4'), 161,07 (C-4"'), 159,26 (C-2), 158,26 (C-9), 146,52 (CH- $\beta$ ) 135,22 (C-3), 132,19 (CH-2'/CH-6'), 131,13 (CH-2'”/CH-6'"), 127,03 (C-1'”),
122,64 (C-1'), 116,73 (CH-3'”/CH-5'”'), 115,99 (CH-3'/CH-5'), $114,70(\mathrm{CH}-\alpha), 105,53$ (C-10), 104,02 (CH-1"), 99,93 (CH-6), 94.85 (CH-8), 77,96 (CH-3"), 75,71 (CH-2", CH-5"), 71,67 (CH-4”), 64,37 ( $\left.\mathrm{CH}_{2}-6 "\right)$

Luteolina 7-O- $\beta$-D-glicopiranosídeo (cinarosídeo, 10). Sólido cristalino. $\mathrm{RMN}{ }^{1} \mathrm{H}\left[200 \mathrm{MHz}, \mathrm{CDCl}_{3}, \delta(\mathrm{ppm}), J(\mathrm{~Hz})\right]: 7,43(d, 2 \mathrm{~Hz}$, H-2'), 6,92 (d, 8,9 Hz, H-5'), 6,78 ( $d, 1,8 \mathrm{~Hz}, \mathrm{H}-8), 6.74$ ( $s, \mathrm{H}-3)$, 6,44 ( $m$, H-6'), 6,43 (d, 1,8 Hz, H-6), 5,07 (d, 7,2 Hz, H-1"), 3,60 ( $m$, H-6"), 3,40 (m, H-3", H-6"), 3,23 (m, H-2"), 3,22 (m, H-5"). $\mathrm{RMN}{ }^{13} \mathrm{C}\left(50 \mathrm{MHz}, \mathrm{CDCl}_{3}, \delta\right.$ (ppm)]: 181,98 (C-4), 164,56 (C-2), 163,01 (C-7), 161,17 (C-5), 157,01 (C-9), 150,09 (C-4'), 145,90 (C-3'), 121,34 (C-1'), 119,20 (CH-6'), 116,33 (CH-5'), 113,66 (CH2'), 105,39 (C-10), 103,16 (CH-3), 99,92 (CH-1"), 99,57 (CH-6), 94,81 (CH-8), 77,20 (CH-3"), 76,44 (CH-2"), 73,17 (CH-5”), 69,58 (CH-4"), 60,64 ( $\left.\mathrm{CH}_{2}-6 "\right)$.

Tabela 1. Dados* $\left(\delta\right.$ e $J \mathrm{~Hz}$, em $\left.\mathrm{CDCl}_{3}\right)$ de $\mathrm{RMN}{ }^{1} \mathrm{H}(500 \mathrm{MHz})$ e ${ }^{13} \mathrm{C}(125 \mathrm{MHz})$ para $17^{3}$-etoxifaeoforbídeo a (2)

\begin{tabular}{|c|c|c|c|c|c|}
\hline & ${ }^{1} \mathrm{H} \mathrm{x}{ }^{13} \mathrm{C}-\mathrm{HMQC}$ & & ${ }_{2}{ }^{1} \mathrm{H}$ & $\mathrm{BC}_{3}$ & 1H-1H-COSY \\
\hline $\mathrm{H}$ & $\delta_{\mathrm{H}}^{\mathrm{CH}}$ & $\delta_{\mathrm{C}}$ & ${ }^{2} J_{\mathrm{CH}}$ & $3_{\mathrm{CH}}$ & \\
\hline 1 & - & 141,98 & & & \\
\hline 2 & - & 131,78 & & & \\
\hline $2^{1}$ & $3,37(\mathrm{~s})$ & 12,06 & $\mathrm{C}-2$ & $\mathrm{C}-1 ; \mathrm{C}-3$ & \\
\hline 3 & - & 136,16 & & & \\
\hline $3^{1}(\mathrm{Ha})$ & $7,93(\mathrm{dd}, J=17,8$ e 11,6$)$ & 128,91 & & & \\
\hline $3^{2}(\mathrm{Hb})$ & $6,14(\mathrm{dd}, J=11,6$ e 1,6$)$ & 122,72 & & & \\
\hline$(\mathrm{Hc})$ & $6,24(\mathrm{dd}, J=17,8$ e 1,6$)$ & & & $\mathrm{C}-3$ & \\
\hline 4 & - & 136,40 & & & \\
\hline 5 & $9,30(\mathrm{~s})$ & 97,39 & $\mathrm{C}-4$ & & \\
\hline 6 & - & 155,55 & & & \\
\hline 7 & - & 136,05 & & & \\
\hline $7^{1}$ & $3,16(\mathrm{~s})$ & 11,11 & $\mathrm{C}-7$ & C-6; C-8 & \\
\hline 8 & - & 145,09 & & & \\
\hline $8^{1}$ & 3,64 (m) & 19,32 & & & $\mathrm{H}-8^{2}$ \\
\hline $8^{2}$ & $1,65(\mathrm{t}, J=7,6)$ & 17,37 & $\mathrm{C}-8^{1}$ & $\mathrm{C}-8$ & \\
\hline 9 & - & 150,86 & & & \\
\hline 10 & $9,45(\mathrm{~s})$ & 104,32 & C-9 & C-8; C-12 & \\
\hline 11 & - & 137,83 & & & \\
\hline 12 & - & 128,80 & & & \\
\hline $12^{1}$ & $3,65(\mathrm{~s})$ & 12,06 & & C-11; C-13 & \\
\hline 13 & - & 128,80 & & & \\
\hline $13^{1}$ & - & 189,66 & & & \\
\hline $13^{2}$ & $6,25(\mathrm{~s})$ & 64,66 & $\mathrm{C}-13^{1} ; \mathrm{C}-15$ & & \\
\hline $13^{3}$ & - & 172,95 & & & \\
\hline $13^{4}$ & 3,87 (s) & 52,88 & & & \\
\hline 14 & - & 149,59 & & & \\
\hline 15 & - & 105,10 & & & \\
\hline 16 & - & 161,19 & & & \\
\hline 17 & 4,19 (m) & 51,05 & & & \\
\hline $17^{1}$ & $1,11(\mathrm{~m})$ & 29,76 & & & \\
\hline $17^{2}$ & $2,0-2,2(\mathrm{~m})$ & 31,16 & & & \\
\hline $17^{3}$ & - & 172,19 & & & \\
\hline $17^{4}$ & $3,99(\mathrm{~m})$ & 60,49 & & & $\mathrm{CH}_{3}-17^{5}$ \\
\hline $17^{5}$ & $1,09(\mathrm{t}, J=7,0)$ & 14,04 & C- $-17^{4}$ & & \\
\hline 18 & $4,44(\mathrm{~m})$ & 50,05 & & & $\mathrm{CH}_{3}-18^{1}, \mathrm{H}-17$ \\
\hline $18^{1}$ & $1,79(\mathrm{~d}, J=7,4)$ & 23,94 & $\mathrm{C}-17$ & & \\
\hline 19 & - & 169,60 & & & \\
\hline 20 & $8,53(\mathrm{~s})$ & 93,06 & C-19 & $\mathrm{C}-2$ & \\
\hline
\end{tabular}

*Espectros 2D de correlação ho2D de correlação homonuclear $\left({ }^{1} \mathrm{H}-{ }^{1} \mathrm{H}-\mathrm{COSY}\right.$ e NOESY) e heteronuclear $\left({ }^{1} \mathrm{H}-{ }^{13} \mathrm{C}\right.$ HMBC) foram também utilizados na interpretação destes dados. A análise dos espectros de RMN ${ }^{13} \mathrm{C}$ HBBD e APT foi usada para identificar os sinais dos átomos de carbono $\mathrm{C}, \mathrm{CH}, \mathrm{CH}_{2}$ e $\mathrm{CH}_{3}$. 


\section{Atividade antioxidante}

A atividade sequiestradora de radical livre para os extratos EtOH, hexânico, AcOEt e BuOH foi determinada utilizando o teste com o $\mathrm{DPPH}$, usando uma série de diluições, misturando-se $5 \mathrm{~mL}$ de solução de DPPH (100 $\mu \mathrm{M}$ em EtOH) com quantidades apropriadas dos extratos (concentrações variando entre 24,0-143,0 $\mu \mathrm{g} / \mathrm{mL}$ ). Após 30 min, a quantidade dos radicais de DPPH foi registrada em UVVis no comprimento de onda $517 \mathrm{~nm}$. O teste foi realizado em triplicata. Foram utilizados como padrão o ácido ascórbico $\left(\mathrm{CE}_{50}=\right.$ $14,08 \mu \mathrm{M}$ e o BHT $\left(\mathrm{CE}_{50}=20,26 \mu \mathrm{M}\right)$. A eficiência antiradicalar foi estabelecida utilizando a análise de regressão linear no intervalo de confiança de $95 \%(\mathrm{P}<0,05)$ obtido pelo programa de estatística GraphPad Prism 4.0. Os resultados foram expressos através do valor da $\mathrm{CE}_{50}$, que representa a concentração da amostra necessária para sequiestrar 50\% dos radicais de DPPH (Tabela 2).

\section{RESULTADOS E DISCUSSÃO}

$\mathrm{O}$ espectro de $\mathrm{RMN}{ }^{1} \mathrm{H}$ de $\mathbf{1}$ mostrou presença de dois dubletos em $\delta_{\mathrm{H}} 6,38$ e $\delta 6,47$, referentes aos hidrogênios 6 e 8 , respectivamente, normalmente decorrentes de substituição por funções oxigenadas nos carbonos 5 e 7 em espectros de flavonas ${ }^{19}$. Absorções para três metoxilas aromáticas foram observadas em $\delta_{\mathrm{H}} 3,88, \delta$ 3,90 e $\delta 4,00$. Uma absorção em campo baixo localizada em $\delta_{H}$ 12,66 corresponde ao valor típico de hidroxila em ponte com carbonila, normalmente observada em espectros de hidrogênio de flavonóides ${ }^{20}$. A presença de um sistema $A B X$ foi deduzida por um conjunto de sinais em $\delta_{\mathrm{H}} 7,69(\mathrm{dd}, J=8,4$ e 1,9 Hz), $\delta$ 7,06 (d, $J=8,4$ $\mathrm{Hz}$ ) e $\delta 7,72$ (d, $J=1,9 \mathrm{~Hz}$ ), sugerindo substituições nos carbonos 3' e 4' do anel B de 1. As posições dos grupos metoxílicos foram estabelecidas pelo espectro HMBC, que mostrou interações a três ligações das absorções em $\delta_{\mathrm{H}} 3,88\left(\mathrm{CH}_{3} \mathrm{O}-3\right) \operatorname{com} \delta_{\mathrm{C}} 139,36(\mathrm{C}-3)$, $\delta_{\mathrm{H}} 3,90\left(\mathrm{C}_{\underline{3}} \mathrm{O}-7\right) \operatorname{com} \delta_{\mathrm{C}} 165,85(\mathrm{C}-7)$ e $\delta_{\mathrm{H}} 4,00\left(\mathrm{C}_{\underline{3}} \mathrm{O}-3\right.$ ') com $\delta_{\mathrm{C}}$ $146,74\left(\mathrm{C}-3^{3}\right)$. Os dados espectrais de 1 foram também comparados com a literatura ${ }^{21}$, permitindo identificá-la como 5,7-diidroxi3,7-3'-trimetoxiflavona, conhecida como pachypodol (1).

$\mathrm{O}$ espectro de IV de 2 mostrou bandas de absorção em $v_{\max }$ $3397 \mathrm{~cm}^{-1}$, referente à deformação axial de $\mathrm{N}-\mathrm{H}, v_{\max } 1344 \mathrm{~cm}^{-1}$, condizente com estiramento de $\mathrm{C}-\mathrm{N}$, e $1612 \mathrm{~cm}^{-1}$, relativo à deformação de ligação dupla em sistemas conjugados, sugerindo a presença de núcleo porfirínico. As bandas em $v_{\max } 1731$ e $1695 \mathrm{~cm}^{-1}$ foram atribuídas a grupos carbonílicos não conjugado e conjugado, respectivamente ${ }^{22}$. O espectro de massas de $\mathbf{2}$ revelou o pico do íon molecular em $\mathrm{m} / \mathrm{z} 621$ [ $\left.\mathrm{M}^{+}+1\right]$, que permitiu a dedução da fórmula molecular como $\mathrm{C}_{37} \mathrm{H}_{40} \mathrm{~N}_{4} \mathrm{O}_{5}$.

$\mathrm{O}$ espectro de $\mathrm{RMN}{ }^{1} \mathrm{H}$ revelou absorções para um grupo de hidrogênios vinílicos em $\delta_{\mathrm{H}} 7,93(d d, J=17,8$ e 11,6 Hz), 6,24 ( $d d$, $J=17,8$ e $1,6 \mathrm{~Hz}), 6,14(d d, J=11,6$ e $1,6 \mathrm{~Hz})$, três metilas olefínicas em $\delta_{\mathrm{H}} 3,37,3,16$ e 3,65 e três hidrogênios olefínicos em $\delta_{\mathrm{H}} 9,30$, 9,45 e 8,53 , estes últimos condizentes com absorções dos hidrogênios 5,10 e 20 do núcleo porfirínico das feofitinas ${ }^{23,24}$. Adicionalmente, observou-se um conjunto de deslocamentos químicos para hidrogênios metoxílicos em $\delta_{\mathrm{H}} 3,87$ e etoxílicos em $\delta_{\mathrm{H}} 3,93(\mathrm{~m})$ e $1,09(t, J=7,0 \mathrm{~Hz})$. Através do espectro de HMBC tornou-se possível estabelecer as posições dos grupos substituintes no anel porfirínico. Este espectro mostrou correlação a três ligações entre os sinais em $\delta_{\mathrm{H}} 6,24$ e $6,14\left(\underline{2 \mathrm{H}}-3^{2}\right) \operatorname{com} \delta_{\mathrm{C}} 136,16(\mathrm{C}-3)$ e ainda entre $\mathrm{CH}_{3}-8^{2}\left(\delta_{\mathrm{H}} 1,65\right)$ com o carbono $8\left(\delta_{\mathrm{C}} 145,09\right)$, permitindo definir as posições 3 e 8 para os grupos vinúlico e etílico, respectivamente. Os assinalamentos referentes aos hidrogênios e grupos metilícos olefínicos foram feitos também através do espectro HMBC com base nas correlações seguintes: $\mathrm{CH}_{3}-2^{1}\left(\delta_{\mathrm{H}} 3,37\right) \operatorname{com} \delta_{\mathrm{C}} 141,98$
$(\mathrm{C}-1)\left({ }^{2} J\right)$ e $\delta_{\mathrm{C}} 136,16(\mathrm{C}-3)\left({ }^{3} J\right) ; \mathrm{CH}_{3}-7^{1}\left(\delta_{\mathrm{H}} 3,16\right) \operatorname{com} \delta_{\mathrm{C}} 136,05$ $\left(\mathrm{C}-7,{ }^{2} J\right)$ e $\mathrm{CH}_{3}-12^{1}\left(\delta_{\mathrm{H}} 3,65\right)$ com $\delta_{\mathrm{C}} 137,83\left(\mathrm{C}-11,{ }^{3} J\right)$. O espectro de NOESY permitiu estabelecer a estereoquímica dos carbonos $17,18^{1}$ e $13^{2}$ através do acoplamento espacial entre as absorções em $\delta_{\mathrm{H}} 4,19(\mathrm{H}-17)$ e $1,79\left(\mathrm{CH}_{3}-18^{1}\right)$, de onde se deduziu que $\mathrm{H}-17$ e $\mathrm{CH}_{3}-18^{1}$ encontram-se em configuração $\alpha$ (Figura 2). A análise espectral combinada com os dados da literatura ${ }^{25}$ permitiu identificar a substância 2 como $17^{3}$-etoxifaeoforbídeo.

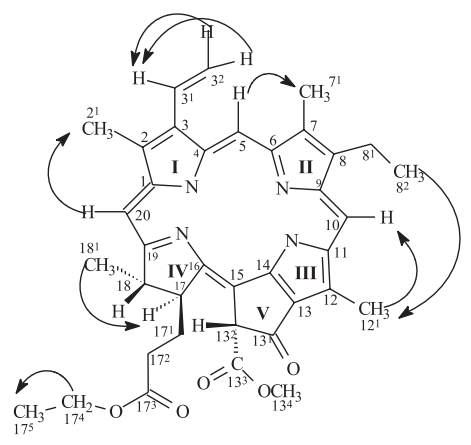

Figura 2. Interações NOE para 2

As estruturas das substâncias 3-9 foram caracterizadas através dos espectros 1D e 2D de $\mathrm{RMN}{ }^{1} \mathrm{H}$ e ${ }^{13} \mathrm{C}$ envolvendo comparação com dados descritos na literatura, permitindo identificá-las como 6,7dimetoxicumariana (escoparona, 3) ${ }^{26,27}$, ácido orto-hidroxibenzóico $(4)^{28}$, sitosterol-3-O- $\beta$-D-glicopiranosídeo $(5)^{29}$ e estigmasterol-3- $O$ $\beta$-D-glicopiranosídeo $(\mathbf{6})^{29}, 5,7,4^{\prime}$-triidroxiflavona apigenina $(7)^{30}$ $5,7,3^{\prime}, 4^{\prime}$ '-tetraidroxiflavona (luteolina, 8) ${ }^{21}$ e canferol-3-O- $\beta$-D- $(6$ 'E-p-cumaroil) glicopiranosídeo tilirosídeo $(\mathbf{9})^{31}$.

Os espectros de $\mathrm{RMN}{ }^{1} \mathrm{H}$ e ${ }^{13} \mathrm{C}$ da substância $\mathbf{1 0}$ mostraram absorções compatíveis com um flavonóide com o padrão de substituição da luteolina (8). O espectro de $\mathrm{RMN}{ }^{1} \mathrm{H}$ apresentou também sinais para uma molécula osídica, caracterizada pelos multipletos entre $\delta_{\mathrm{H}} 2,90-4,00$ e um dubleto em $\delta_{\mathrm{H}} 5,07(J=7,20 \mathrm{~Hz})$ atribuído a hidrogênio anomérico, sugerindo a presença de uma unidade glicopiranozílica com configuração $\beta$. O espectro de NOESY de 10 mostrou acoplamento espacial entre o hidrogênio anomérico 1" $\left(\delta_{\mathrm{H}} 5,06\right)$ com os hidrogênios H-6 $\left(\delta_{\mathrm{H}} 6,42\right)$ e H-8 $\left(\delta_{\mathrm{H}} 6,78\right)$, permitindo, conseqüentemente, localizar a $O$-glicosilação na posição 7 do esqueleto da luteolina. Este espectro mostrou ainda as interações espaciais entre os hidrogênios H-3 $\left(\delta_{H} 6,74\right)$ e H-6' $\left(\delta_{H} 7,43\right)$, sugerindo a ausência de substituinite no átomo de carbono $\mathrm{C}-3$ e contribuindo para a localização dos demais substituintes na molécula (Figura 3). Os dados espectrais foram também comparados com
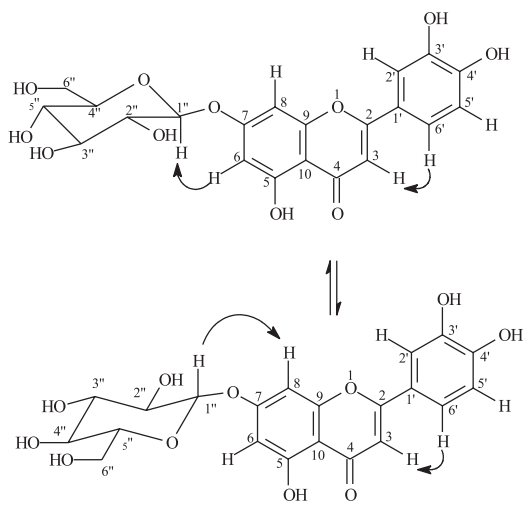

Figura 3. Interações NOE para 10 
valores registrados na literatura ${ }^{32}$, permitindo caracterizar a estrutura da luteolina 7-O- $\beta$-D-glicopiranosídeo (cinarosídeo, 10).

\section{Atividade antioxidante}

A atividade antioxidante utilizando o radical DPPH tem sido muito utilizada para verificar a capacidade seqüestradora de radicais livres de muitos produtos naturais ${ }^{33-35}$.

Como mostrado na Figura 4 e Tabela 2, a atividade antiradicalar de diferentes extratos de Sida galheirensis foi determinada utilizando o radical livre DPPH. Os valores foram expressos através de $\mathrm{EC}_{50}$. $\mathrm{O}$ extrato AcOEt revelou-se o mais ativo. A atividade diminuiu na seguinte ordem: ácido ascórbico $>\mathrm{BHT}>\mathrm{AcOEt}>\mathrm{BuOH}>\mathrm{EtOH}>$ $\mathrm{CHCl}_{3}>$ hexânico. $\mathrm{O}$ alto valor de $\mathrm{EC}_{50}$ para o extrato hexânico mostrou uma menor capacidade de sequiestrar os radicais livres. A alta atividade seqüestradora de radicais livres para o AcOEt pode ser justificada pela presença de pelo menos dois dos flavonóides. Em trabalhos anteriores foi verificada a atividade antiradicalar, utilizando o DPPH, para os flavonóides luteolina ${ }^{36}$ e tilirosídeo ${ }^{36}$, sendo a flavona apigenina ${ }^{37}$ inativa frente a este radical.

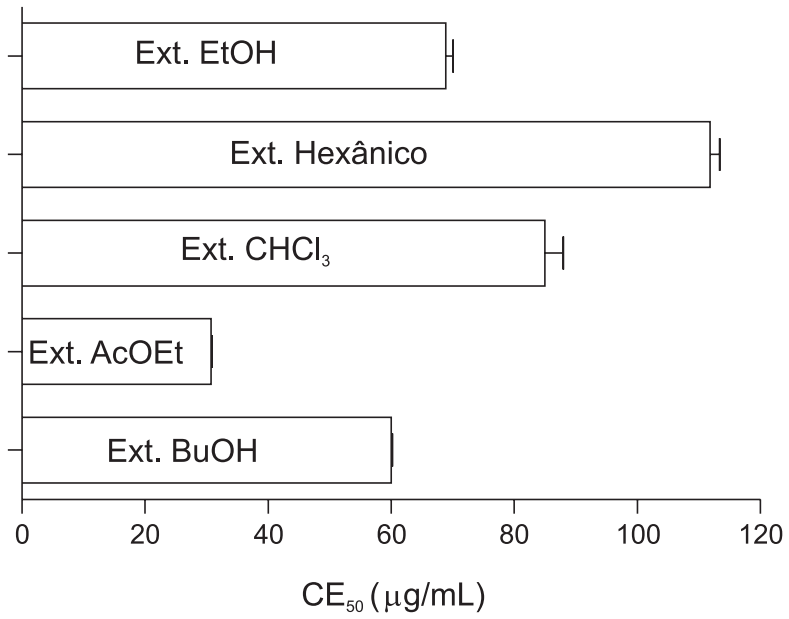

Figura 4. Comparação dos valores de $C E_{50}$ dos diferentes extratos obtidos de Sida galheirensis. Os resultados são expressos como a média $\pm \operatorname{SEM}(\mathrm{n}=3)$

Tabela 2. Atividade antioxidante dos diferentes extratos de Sida galheirensis utilizando o radical livre DPPH

\begin{tabular}{lc}
\hline Extrato & $\mathrm{CE}_{50}(\mu \mathrm{g} / \mathrm{mL}) \pm \mathrm{SEM}^{\mathrm{a}}$ \\
\hline EtOH & $* 68,9 \pm 1,2$ \\
$\mathrm{Hexânico}$ & $111,8 \pm 1,6$ \\
$\mathrm{CHCl}_{3}$ & $85,0 \pm 3,0$ \\
$\mathrm{AcOEt}^{\mathrm{BuOH}}$ & $30,8 \pm 0,1$ \\
\end{tabular}

*Média \pm SEM $(n=3)$; ${ }^{\text {a Concentração suficiente para obter } 50 \%}$ da capacidade máxima de seqüestrar os radicais livres (descrito em materiais e métodos). Os valores de $\mathrm{CE}_{50}$ foram calculados pela reta da regressão linear.

\section{AGRADECIMENTOS}

Ao PRONEX/CNPq e FAPERJ pela bolsa (CNPq) e pelo suporte financeiro, ao CENAUREM/UFC pela obtenção dos espec- tros de 500 e 125 MHz, à Profa M. de F. Agra (LTF/DCF/UFPB) pela identificação botânica e a V. C. da Costa (NPPN/LTF/UFPB) pela obtenção dos espectros de 200 e $50 \mathrm{MHz}$.

\section{REFERÊNCIAS}

1. Baracho, G. S.: Dissertação de Mestrado, Universidade Federal da Paraíba, Brasil, 1998.

2. Otero, R.; Nuñez, V.; Barona, J.; Fonnegra, R.; Jiménez, S. L.; Osorio, R. G.; Saldarriaga, M.; Díaz, A.; Journal of Ethnopharmacology 2000, 73, 233.

3. Franzotti, E. M.; Santos, C. V. F.; Rodrigues, H. M. S. L.; Mourão, R. H. V.; Andrade, M. R.; Antoniolli, A. R.; J. Ethnopharmacol. 2000, 72, 273.

4. Venkatesh, S.; Reddy, S. R.; Suresh, B.; Ressy, B. M.; Ramesh, M.; J. Ethnopharmacol. 1999, 67, 229.

5. Rao, R. E.; Dixit, V. K.; Varma, K. C.; J. Am. Oil Chem. Soc. 1973, 50, 168.

6. Pande, C. S.; Tewari, J. D.; J. Oil Technol. Ass India 1960, 29, 16.

7. Bhatt, D. J. J.; Baxi, A. J.; Parikh, A. R.; J. Indian Chem. Soc. 1983, 60, 98.

8. Husain, S.; Babu, M.; Ahmad, M. U.; Ansari, A. A.; Osman, S. M.; Fette Seifen Anstrichm 1980, 82, 29.

9. Ahmad, M. U.; Husain, S. K.; Ahmad, M.; Osman, S. M.; Subarro, R.; J. Am. Oil Chem. Soc. 1976, 53, 698.

10. Goyal, M. M.; Rani, K. K. J.; Indian Chem. Soc. 1988, 65, 74.

11. Chouhan, U. K.; Skukla, R. N.; J. Sci. Res. 1984, 6, 49.

12. Matlawska, I.; Herba Pol. 1990, 36, 65.

13. Ligai, L. V.; Bandyukova, V. A.; Chem. Nat. Compd. 1990, 26, 221.

14. Pyrek, J.; Chari, M.; Abstracts of $24^{\text {th }}$ Annual Meeting American Society of Pharmacognosy, Mississippi, USA, 1983.

15. Prakash, A.; Varma, R. K.; Ghosal, S.; Planta Med. 1981, 43, 384.

16. Ames, B. N.; Shigenaga, M. K.; Hagen, T. M.; Proc. Natl. Acad. Sci. U. S. A. 1993, 90, 79 .

17. Noguchi, C.; Niki, E.; Free Radical Biol. Medi. 2000, 28, 1538.

18. Visioli, F.; Borsani, L.; Galli, C.; Cardiovasc. Res. 2000, 47, 419.

19. Harborne, J. B.; The Flavonoids - Advances in Research since 1986, Cahpman \& Hall: London, 1994.

20. Kinoshita, T.; Firman, K.; Phytochemistry 1996, 42, 1207.

21. Agrawal, P. K.; Carbon-13 NMR of Flavonoids: Studies in Organic Chemistry 39, Lucknov Elsevier: India, 1989.

22. Silverstein, R. M.; Bassler, G. C.; Morrill, T. C.; Identificação Espectrométrica de Compostos Orgâncos, Ed. Guanabara Koogan S.A.: Rio de Janeiro, 1994.

23. Matsuo, A.; Ono, K.; Hamasaki, K.; Nozaki, H.; Phytochemistry 1996, 42, 427.

24. Duan, H.; Takaish, Y.; Momota, H.; Ohmoto, Y.; Taki, T.; Phytochemistry 2002, 59, 85 .

25. Schwikkard, S. L.; Mulholland, A.; Phytochemistry 1998, 49, 2391.

26. Tsukamoto, H.; Hisada, S.; Nishibe, S.; Roux, D. G.; Rourke, J. P.; Phytochemistry 1984, 23, 699.

27. Inuma, M.; Ohyama, M.; Tanaka, T.; Mizuno, M.; Hong, S. K.; Phytochemistry 1993, 33, 1241.

28. Kwon, Y.; J. Mol. Struct. (Teochem) 2000, 532, 227.

29. Kojima, H.; Sato, N.; Hatano, A.; Ogura, H.; Phytochemistry 1990, 29, 2351.

30. Shen, C. C.; Chang, Y. S.; Ho, L. K.; Phytochemistry 1993, 34, 843.

31. Kaouadji, M.; Doucouré, A.; Mariotte, A. M.; Chulia, A. J.; Phytochemistry 1990, 29, 1283.

32. Markham, K. R.; Ternai, B.; Stanley, R.; Geiger, H.; Mabry, T. J.; Tetrahedron 1978, 34, 1389.

33. Ahmad, R.; Ali, A. M.; Israf, D. A.; Ismail, N. H.; Shaari, K.; Lajis, N. H.; Life Sci. 2005, 76, 1953.

34. Nessa, F.; Ismail, Z.; Mohamed, N.; Haris, M. R. H. M.; Food Chem. 2004, 8, 243.

35. Kogure, K.; Yamacuchi, I.; Tokumura, A.; Kondou, K.; Tanaka, N.; Takaishi, Y.; Fukuzawa, K.; Phytomedicine 2004, 11, 645.

36. Sala, A.; Recio, M. C.; Schinella, G. R.; Manez, S.; Giner, R. M.; CerdaNicolas, M.; Rios. J. L; Eur. J. Pharmacol. 2003, 53, 461.

37. Furusawa, M.; Tanaka, T.; Ito, T.; Nishikawa, A.; Yamazaki, N.; Nakaya, K.; Marsuura, N.; Tsuchiya, H.; Nagayama, M.; Inuma, M.; J. Health Sci. 2005, 51, 376. 